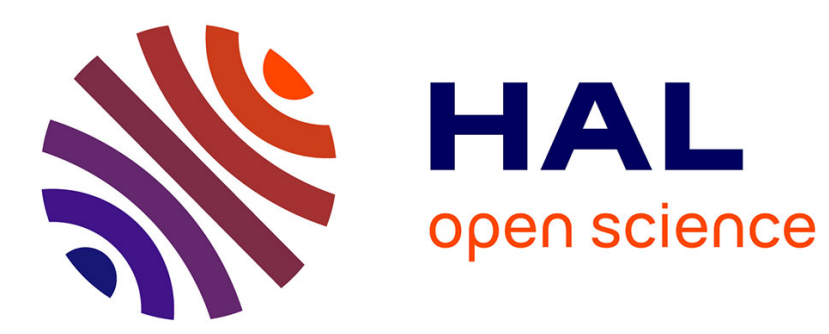

\title{
In-Situ Electrical Biasing of Electrically Connected TEM Lamellae with Embedded Nanodevices
}

Maria Brodovoi, Kilian Gruel, Lucas Chapuis, Aurélien Masseboeuf, Cécile Marcelot, Martin Hÿtch, Frédéric Lorut, Christophe Gatel

\section{- To cite this version:}

Maria Brodovoi, Kilian Gruel, Lucas Chapuis, Aurélien Masseboeuf, Cécile Marcelot, et al.. In-Situ Electrical Biasing of Electrically Connected TEM Lamellae with Embedded Nanodevices. ISTFA 2021, Oct 2021, Phoenix, France. pp.190-195, 10.31399/asm.cp.istfa2021p0190 . hal-03412037

\section{HAL Id: hal-03412037 https://hal.science/hal-03412037}

Submitted on 23 Nov 2021

HAL is a multi-disciplinary open access archive for the deposit and dissemination of scientific research documents, whether they are published or not. The documents may come from teaching and research institutions in France or abroad, or from public or private research centers.
L'archive ouverte pluridisciplinaire HAL, est destinée au dépôt et à la diffusion de documents scientifiques de niveau recherche, publiés ou non, émanant des établissements d'enseignement et de recherche français ou étrangers, des laboratoires publics ou privés. 


\title{
In-situ electrical biasing of electrically connected TEM lamellae with embedded nanodevices
}

\author{
Maria Brodovoi'1,2, Kilian Gruel', Lucas Chapuis ${ }^{1}$, Aurélien Masseboeuf', Cécile Marcelot ${ }^{1}$, Martin Hÿtch ${ }^{1}$, Frédéric \\ Lorut ${ }^{2}$, Christophe Gatel ${ }^{1}$ \\ ${ }^{1}$ CEMES-CNRS, Toulouse, France \\ 2STMicroelectronics, Crolles, France
}

\begin{abstract}
In response to a continually rising demand for high performance and low-cost devices, and equally driven by competitivity, the microelectronics industry excels in meeting innovation challenges and further miniaturizing products. However, device shrinkage and the increasing complexity of device architecture require local quantitative studies. In this paper, we demonstrate with a case study on a nanocapacitor, the capability of transmission electron microscopy in electron holography mode to be a unique in-situ technique for mapping electric fields and charge distributions on a single device.
\end{abstract}

\section{Introduction}

In recent years, there has been an increased interest in understanding local behavior and physical phenomena of electronic devices at the $\mathrm{nm}$-scale. One of the readily available and appropriate tools for studying a wide variety of materials and nanosystems is Transmission Electron Microscopy (TEM). Its broad sensitivity, coupled to progress in aberrationcorrection, allows modern TEMs to reach spatial resolutions less than $50 \mathrm{pm}$. Besides conventional morphology and local structural and chemical studies, TEM investigations based on off-axis electron holography can also determine the electromagnetic [1-3] and deformation fields [4] at the nanometer scale.

Even though analytical TEM routinely contributes to failure analysis of semiconductor devices [5], improvement or development of new devices require a deeper understanding of local properties when in operation. Operando TEM experiments, i.e., in-situ TEM biasing of electronic devices, are however rarely attempted [6-9]. On the other hand, having access to local electric fields and electric charge distribution mapped at the nanometer scale across a single device in operation would address critical information for future developments or technology design improvements and optimization. The resulting information can also be beneficial to failure analysis, where very precise high-resolution local observations of nanodevices in operation could be used to observe isolated defects or abnormalities in a single region.

This paper proposes a methodology to study electronic devices under real operation conditions by coupling advanced focusedion beam (FIB) sample preparation and off-axis electron holography. Additional finite element modeling (FEM) will allow the experimental results to be compared to numerical simulations including factors like specimen geometry, FIBinduced damage, stray fields, and charging effect from electron beam interactions.

\section{Experimental}

\section{Sample preparation}

Experiments were carried out on a MIM (Metal-InsulatorMetal) matrix of unitary nanocapacitors manufactured by STMicroelectronics via their $28 \mathrm{~nm}$ FD-SOI (Fully Depleted Silicon On Insulator) process. A capacitor is a passive electrical device used for energy storage and delivery in analog and RF circuit applications. It is embedded in the intermediate backend dielectric layers and is composed of a thin layer of dielectric material sandwiched between two electrically separated metal electrodes. Our device consisted of a common $35 \mathrm{~nm}$ titanium nitride (TiN) bottom electrode, a thin $11.5 \mathrm{~nm}$ tantalum pentoxide $\left(\mathrm{Ta}_{2} \mathrm{O}_{5}\right)$ insulator and $55 \mathrm{~nm}$ TiN top electrode (Figure 1).
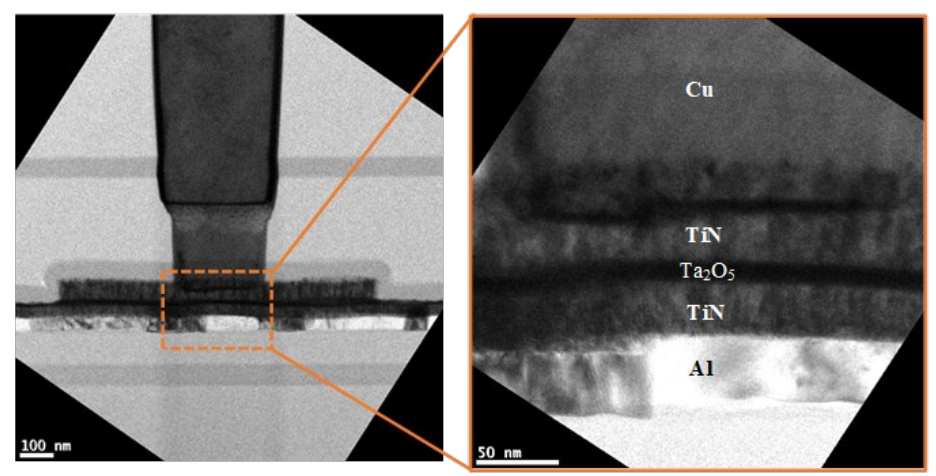

Figure 1: TEM image of MIM nanocapacitor stack.

A specific sample preparation, combining a TEM lamella preparation flow and a FIB-Edit capability, is required to bring electrical stimuli to the device while ensuring its electrical functionality and compatibility with TEM observations. This step is challenging, as the approach and methods for circuit modification and electrical connections must be adapted to the sub- $\mu$ scale. Samples are lifted-out from a fully processed wafer and prepared for electron transparency and in-situ biasing using a ThermoFisher Scientific ${ }^{\mathrm{TM}}$ Helios NanoLab 1200AT DualBeam ${ }^{\mathrm{TM}}$ with a Gallium liquid-metal ion source. The 
equipment includes a multiple line gas injection delivery system (MultiChem) and a gas injection system (GIS), both allowing ion and electron beam-induced deposition and selective etching for effective and high-rate circuit modification.

A conductive surface is essential for optimal charge evacuation during sample navigation and preparation; therefore, a thin conductive layer (gold-palladium alloy) is deposited on the specimen's surface, before any further steps, to ensure charge evacuation. The sample is then grounded by opening the pad (Fig. 2.a) and connecting the top surface to the grounded $\mathrm{Si}$ substrate: the charge from scanning electron beam (SEM) will be drained off to the ground during sample preparation.

Capacitors are susceptible to electrostatic discharge (ESD) damage; therefore, it is important to prevent the structure from floating during FIB-Edit. This can be done by connecting, in advance, both top and bottom electrodes. This is achieved by milling a line on the top surface and filling it with Pt (Fig. 2.a). This protective layer serves multiple purposes: it will act like a shield and preserve the structure during FIB milling, it will prevent charge accumulation, and it will temporarily bring both electrodes to an equipotential. The sample is then top-down deprocessed using FIB until the layer of interest is reached. $\mathrm{Cu}$ lines which connect the nanocapacitors are now exposed (Fig. 2.b) and once again, top and bottom electrodes are brought to the same potential by depositing a Pt layer (Fig. 2.c).

The extracted sample is directly welded to an electrontransparent $\mathrm{Si}_{3} \mathrm{~N}_{4}$ grid with $\mathrm{Au}$ prepatterned tracks (Fig. 2.e,f). $\mathrm{W}$ depositions are used to ensure electrical connection between the sample and grid. To meet electron holography criteria, the region of interest (here, $\mathrm{Ta}_{2} \mathrm{O}_{5}$ oxide) must be no more than 500 $\mathrm{nm}$ from the vacuum region (Fig. 2.g) where the reference wave will be. The next preparation step consists of separating the top electrode from the bottom one to create two distinct electrical connections for in-situ biasing. Two separation cuts (bottom left and top right) are visible on Fig. 2.h.

A final thinning of the lamella with a low energy ion beam ( 8 or $16 \mathrm{kV}$ ) for minimizing surface damage is performed until a uniform thickness below $100 \mathrm{~nm}$ is reached for electron transparency.
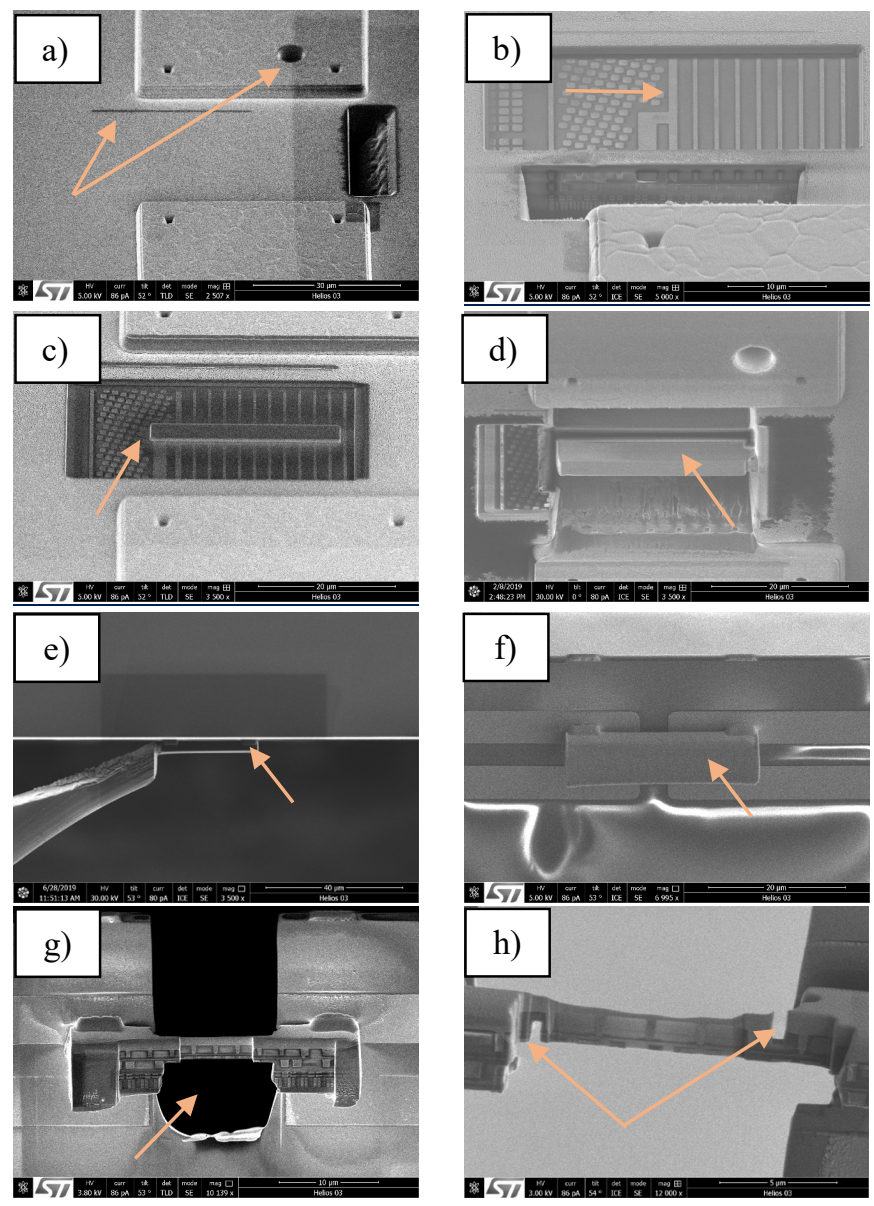

Figure 2: a) Sample grounding \& electrode connections. b) Visible Cu lines after FIB deprocessing. c) Protective Pt layer. d) Sample before lift-out. e) Sample welding to the dedicated TEM grid. f) Chunk welded on the TEM grid. g) TEM grid membrane milling \& free area for $E H$ reference wave. h) Final thinning \& electrodes separation.

\section{TEM experimental setup}

To perform in-situ electrical biasing experiments, a dedicated experimental setup must be implemented that consists of several levels of electrical connections (Fig. 3). These are required to bring electrical stimuli from the external power supply to the device embedded in the TEM lamella. The setup has been optimized to avoid electrostatic discharge (ESD) damage when inserting and observing the prepared device. As described previously, the TEM lamella (Fig. 3.e) is already welded to a grid with electrical contacts (Fig. 3.d) to bias the nanocapacitors. The $\mathrm{Si}_{3} \mathrm{~N}_{4}$ grid is then mounted on a single-tilt Gatan HC3500 TEM holder (Fig. 3.b) equipped with 4 electrical contacts to input/output electrical signals from an external current/voltage generator. The grid is electrically connected to the holder contacts with thin conductive wires via $\mu$-bonding (Fig. 3.c).

Before operando TEM experiments, electrical testing (resistance measurements) is performed on the final connected electron transparent lamella to confirm that the nanocapacitors have not been electrically shorted. It should be pointed out that 
a resistance of several hundreds of ohms is acceptable for subsequent electrical biasing.

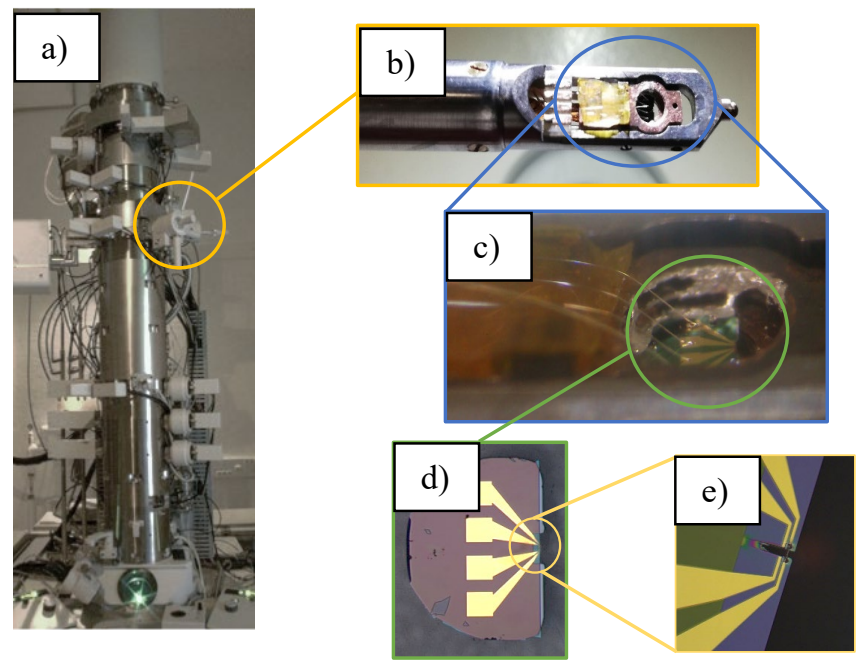

Figure 3: Experimental setup; electrical connectivity levels: ab) TEM microscope - sample holder for electrical biasing via external electrical wiring. b-c) Sample holder-specific TEM grid via $\mu$-bonding. $d$-e) TEM grid-device embedded in the TEM lamella via FIB-assisted conductive layers.

TEM experiments were carried out on the I2TEM-Toulouse microscope, an HF3300-C (Hitachi) TEM specially designed for in-situ electron interferometry experiments. The microscope is equipped with a cold field-emission gun (CFEG) for optimal brightness and coherence, a double stage configuration and 2 post-specimen biprisms. The latest generation of imaging aberration corrector (BCOR from CEOS) allows for on and offaxis aberrations to be corrected in either Lorentz or conventional stage mode allowing wide fields of view and spatial resolution of $0.5 \mathrm{~nm}$ for the Lorentz mode and $87 \mathrm{pm}$ in conventional HRTEM imaging [10]. Completing the setup is a high-speed $4 \mathrm{~K}$ by $4 \mathrm{~K}$ digital camera (OneView, Gatan Inc.).

\section{Electron holography principle}

The principle of off-axis electron holography relies on interfering two parts of a highly coherent electron: the object wave, which interacts with the sample and the surrounding electromagnetic fields, and the reference wave that has not undergone interaction with any field (Figure 4). The resulting interference pattern, called hologram, encodes the phase shift that the electron wave experienced when interacting with the local electromagnetic potentials, mandatory for quantitative mapping of electric fields inside and outside the device [11-12].

The phase change caused by the electrical biasing and recorded in the $x-y$ plane of the hologram is related to the electric potentials in the following way:

$$
\phi(x, y)=c_{E} \int V(x, y, z) d z
$$

where $c_{E}$ is a constant depending on the accelerating voltage of the fast electron and $z$ the direction of the electron path. $V$ is the sum of two contributions:

$$
V=V_{M I P}+V_{E}
$$

with $V_{M I P}$ related to the mean inner potential (MIP) proportional to the atomic number of the encountered atoms along the electron path and $V_{E}$ the electric potential due to the applied bias. As a consequence, the measured phase shift can be separated in two contributions $\phi_{M I P}$ and $\phi_{E}$. Only the latter is of interest in our study and will have to be extracted from the measured phase shift. Fortunately, $\phi_{M I P}$ is independent of the applied bias and can be obtained at $0 \mathrm{~V}$ before being subtracted from the total phase. The integration along the path of the incident electron means that the phase is sensitive to the electric potential within and external to the specimen (i.e., includes the contribution from stray fields).

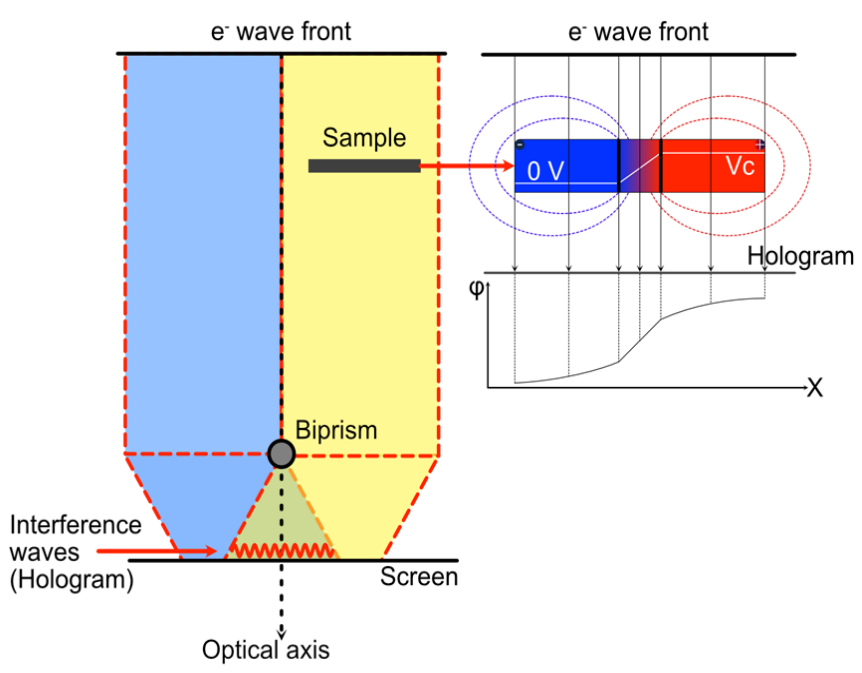

Figure 4: Principle of electron holography and link between potential distribution made by a capacitor and the observed phase.

\section{Results}

\section{Electron holography experiments}

Operando electron holography experiments were performed with an operating voltage of $300 \mathrm{kV}$, Lorentz stage, elliptical illumination and 2 post-specimen biprisms for flexibility in the holographic configurations and to eliminate the Fresnel fringe artefacts [13].

Holograms were acquired during a long exposure time of $120 \mathrm{~s}$ using dynamic automation [14] and $\pi$-shift method [15]. The fringe spacing was set to $1.1 \mathrm{~nm}$ (7 pixels). On-line phase images were displayed with the software package, HoloLive! (HREM Research Inc.), a plug-in for Digital Micrograph (Gatan Inc.). Off-line data processing was carried out with a $5^{\text {th }}$ order Butterworth Fourier-space filter centered on the sideband using routines based on Fourier analysis similar to those developed for the processing of dark-field electron holograms HoloDark 2.0 (HREM Research Inc.) [16]. The numerical mask size was chosen to obtain a spatial resolution of $2 \mathrm{~nm}$ in the final phase images. The projector and camera distortion-induced phase modulations were removed using a reference hologram 
recorded in the field-free vacuum area. The nanocapacitor studied is shown Fig. 5.

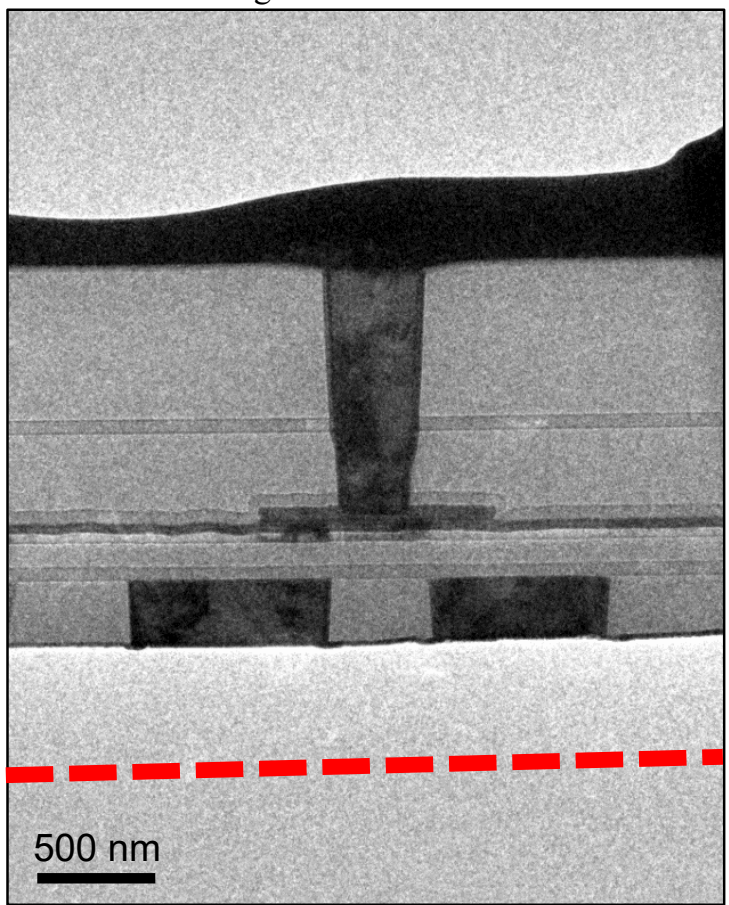

Figure 5: Low magnification TEM micrograph of the studied MIM nanocapacitor. The red dotted line represents the position of the biprism.

For each applied bias, 3 different holograms were recorded for reconstructing the whole field of view. Fig. 6.a and 6.b correspond respectively to the amplitude image and the reference phase image at $0 \mathrm{~V}\left(\phi_{M I P}\right)$ extracted from the holograms. Fig. 6.c and 6.d show the induced electric phase shift at $0.5 \mathrm{~V}$ and $1 \mathrm{~V}$ of applied bias respectively after subtracting the reference phase image at $0 \mathrm{~V}$ : they only contain the electrostatic contribution of excess charges (due to voltage between the electrodes). As expected, the phase shift appears uniform inside the electrodes while a stray field is visible at the edges of the upper electrode due to its finite lateral dimension. The local phase variation located in the $\mathrm{Ta}_{2} \mathrm{O}_{5}$ oxide layer is associated to the electric voltage between the two TiN metal electrodes and consequently to the presence of a strong electric field (Fig. 6.d).

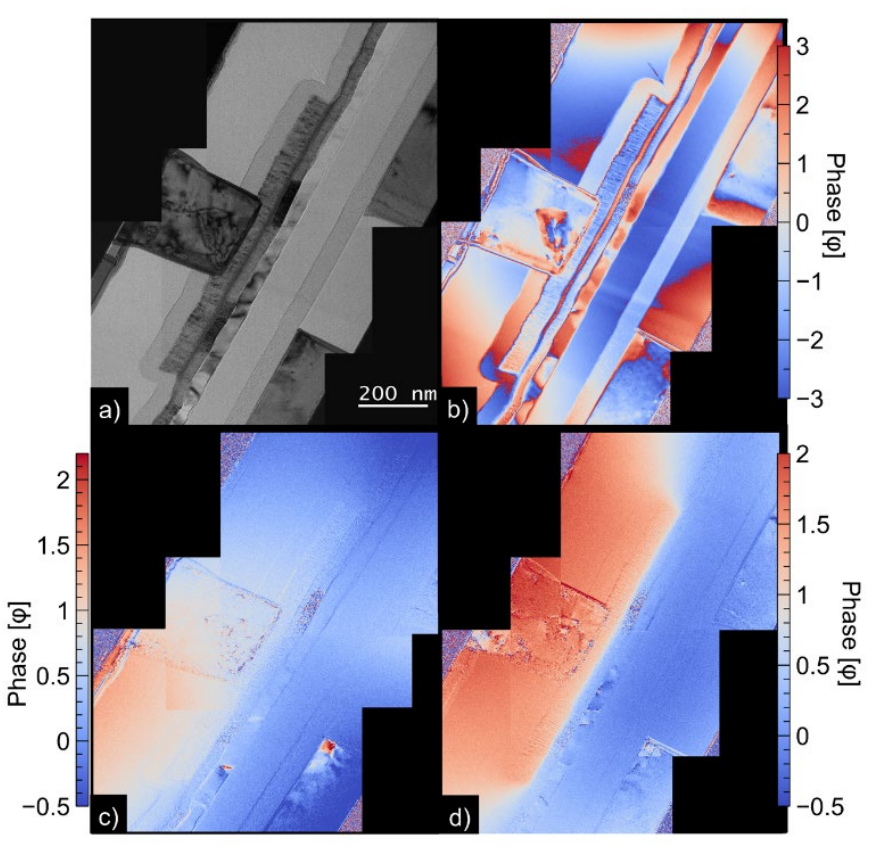

Figure 6: a) Amplitude image of the capacitor C. b) Phase image under $0 \mathrm{~V}$ bias used to remove the mean inner potential contribution. c) Phase image of the induced electrostatic potential under $0.5 \mathrm{~V}$ bias. d) Phase image of the induced electrostatic potential under $1 \mathrm{~V}$ bias.

Three-dimensional (3D) finite element modeling (FEM) was performed with multiphysics software from COMSOL Multiphysics ${ }^{\circledR}$ (v.5.2 and license $\left.\mathrm{N}^{\circ} 1022241\right)$ to have a deeper understanding and quantification of the electric potential distribution as a function of applied bias. The simulations enabled us to assess the impact of the 3D sample geometry and artefacts of preparation, namely FIB-induced sample degradation, on the final device behavior. The FIB-induced preparation artefacts to be considered are the local modification of the conductivity (i.e., conductive damage layers on both sides of the TEM lamella) and the creation of point defects which may modify the electric state of the sample. In addition, modeling allows us to separate the effects of the internal and external/leakage fields.

Regions of different mesh sizes were used to improve the efficiency of the calculations to create a model that sufficiently encompassed the device and surrounding vacuum. The model was surrounded by a layer that approximates an extension of the equations to infinity. Then, the mesh evolves into a free triangular type between the external boundaries and the nanocapacitor. The maximum mesh size in this area is $0.4 \mu \mathrm{m}$. The mesh dynamically adapts in size to meet the effective area of the nanocapacitor with a finer mesh size of about $2 \mathrm{~nm}$ minimum. The model is further divided into two main parts representing two distinct physics and allowing the simulation of conductive and insulating regions: Ohm's law (1) for induction and Gauss' law (2) for electrostatics, respectively. To achieve this, the variable $V$ solved by these two parts must be 
identical

$$
-\nabla \cdot\left(\sigma \nabla V-\overrightarrow{J_{e}}\right)=Q_{j}(1)
$$

both

areas.

$\sigma:$ conductivity $\mathrm{S} / \mathrm{m} ; \overrightarrow{J_{e}}$ : external (injected) current density $\mathrm{A} / \mathrm{m}^{2} ; Q_{j}$ : current source $\mathrm{A} / \mathrm{m}^{3}$.

$$
-\nabla \cdot\left(\varepsilon_{0} \nabla V-\vec{P}\right)=\rho(2)
$$

$\varepsilon_{0}$ : vacuum permittivity $\mathrm{F} / \mathrm{m} ; \vec{P}$ : electric polarization vector $\mathrm{C} / \mathrm{m}^{2} ; \rho:$ space charge density $\mathrm{C} / \mathrm{m}^{3}$.

Fig. 7 shows the comparison between the experimental (Fig. 7.b) result obtained for an applied bias of $1 \mathrm{~V}$ and simulated (Fig. 7.d) phase image. We can see the excellent quantitative agreement, confirmed by the phase profiles extracted through the electrodes and the insulating $\mathrm{Ta}_{2} \mathrm{O}_{5}$ layer (Fig. 7.c). Simulations confirm the important linear variation of the phase shift which occurs in the oxide layer whilst the curvature of the phase in the electrodes caused by the stray field around the sample (see Fig. 4) is well reproduced. However, this agreement has been obtained for a reduced potential drop between the electrodes.
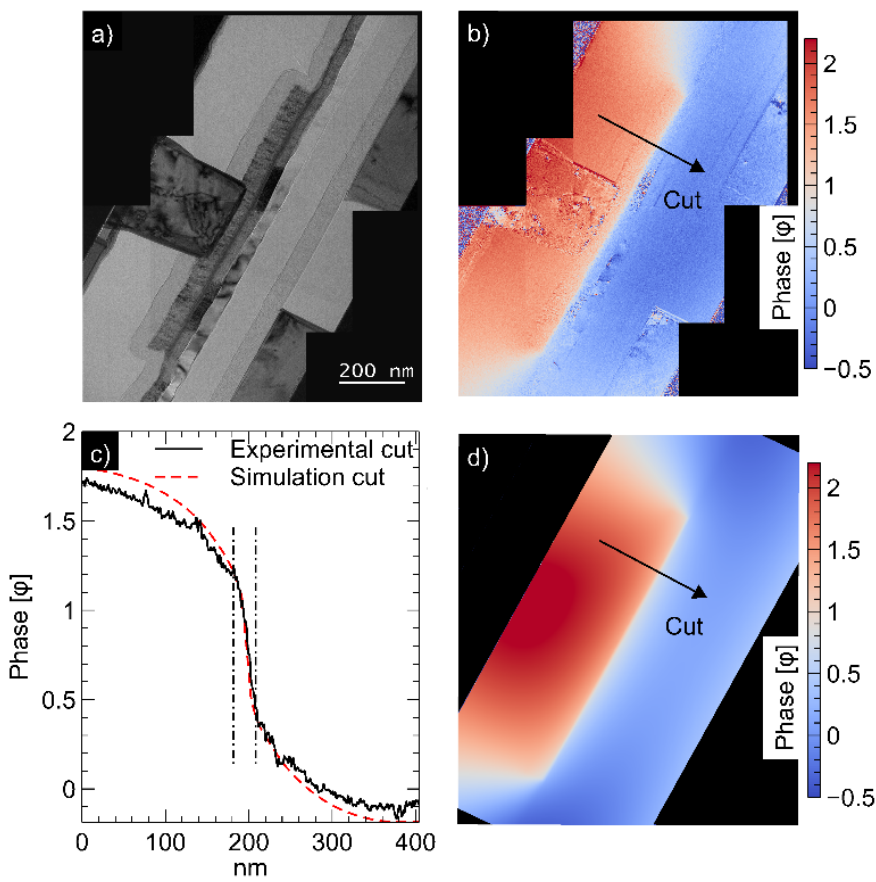

Figure 7: Comparison of experimental and simulated data a) Amplitude image of the studied capacitor. b) Phase image of the same capacitor biased under $1 V$. c) Profiles of the experimental and simulated phase image extracted along the black arrows on the phase images. d) Simulated phase image from FEM.

Since the sample was thinned with a Ga-based focused ion beam, the FEM model also includes conductive layers around the sample. This reduces the potential drop across the dielectric with respect to the applied voltage due to current leakage in the conductive layers. Parameters such as the thickness (due to amorphization on the edges of specimen) and the conductivity of the surface layers were adjusted to produce the best fit with the experimentally measured phase shift (Fig. 7.c). Experimental and theoretical curves may however present discrepancies (like, notable curvature inside the dielectric, related to the evolution of accumulated charge in the oxide) which may need further adjustments by introducing different conditions to the finite element model.

\section{Conclusions}

A set of MIM nanocapacitors have been extracted from production lines and biased for in-situ electrical experiments within the TEM. With the device functioning in real working conditions, operando electron holography allowed the electric potential to be mapped across the active areas and the surrounding stray fields. By comparing the experimental results with FEM modeling, local electrical parameters could be deduced such as electrostatic potential and electric field inside the dielectric layer, capacitance, and surface charge density. The study represents a complete in-situ electrical investigation of a single device under fixed bias and further interpretation of these results will help to enhance sample preparation and minimize its impact on physical parameters modification.

There is no reason that the approach cannot be applied to more complex electronic devices (transistor, phase change memory) by modifying the sample preparation workflow to achieve a successful circuit modification and enable electrical stimulation of the device. We hope that the ability to study local electric fields in targeted devices from production lines will help efforts to improve devices in terms of reliability, speed, and power consumption.

\section{Acknowledgments}

The authors acknowledge the French National Research Agency under the "Investissement d'Avenir" program reference No. ANR-10-EQPX-38-01" and No. 11-IDEX-0002, the "Conseil Regional Midi-Pyrénées" and the European FEDER for financial support within the CPER program. This work was also supported by the French national project IODA (ANR-17CE24-0047). This project (ADVENT - 16ENG06) has received funding from the EMPIR programme co-financed by the Participating States and from the European Union's Horizon 2020 research and innovation programme. The research leading to these results has received funding from the European Union Horizon 2020 research and innovation programme under grant agreement No. 823717 - ESTEEM3.

\section{References}

[1] C. Gatel, B. Warot-Fonrose, N. Bizière, L. A. Rodriguez, D. Reyes, R. Cours, M. Castiella, M.-J. Casanove "Inhomogeneous spatial distribution of the magnetic transition in an iron-rhodium thin film," Nature Communications 8, 15703, 2017. DOI: $10.1038 /$ ncomms 15703 
[2] I.M. Andersen, D. Wolf, L.-A. Rodriguez, A. Lubk, D. Oliveros, C. ban. T. Niermann, U.K. Röler, M. Vasquez, C. Gatel and E. Snoeck "Field tunable three-dimensional magnetic nanotextures in cobalt-nickel nanowires," Physical Review Research 3, 033085 (2021)

DOI: $10.1021 /$ acs.nanolett.1c01087

[3] P.A. Midgley \& R.E. Dunin-Borkowski "Electron tomography and holography in materials science," Nature Materials, vol. 8, pp. 271-280, 2009. DOI: 10.1038/nmat2406

[4] M.J. Hÿtch, F. Houdellier, F. Hüe, and E. Snoeck "Nanoscale holographic interferometry for strain measurements in electronic devices," Nature, vol. 453, pp. 1086-1089, 2008. DOI:10.1038/nature07049

[5] H.J. Engelmann, H. Saage, E. Zschech "Application of analytical TEM for failure analysis of semiconductor device structures," Microelectronics Reliability, vol. 40, no. $8-10$, pp. $1747-1751,2000$. DOI: $10.1016 /$ S00262714(00)00107-4

[6] A.C. Twitchett, R.E. Dunin-Borkowski, P.A. Midgley "Quantitative electron holography of biased semiconductor devices," Phys. Rev. Lett., vol. 88, pp. 238302, 2002. DOI:10.1103/PhysRevLett.88.238302

[7] N. Ikarashi, H. Takeda, K. Yako, and M. Hane "In-situ electron holography of surface potential response to gate voltage application in a sub-30-nm gate-length metaloxide-semiconductor field-effect transistor," Appl. Phys. Lett., vol. 100, pp. 143508, 2012. DOI: $10.1063 / 1.3700723$

[8] L. de Knoop, F. Houdellier, C. Gatel, A. Masseboeuf, M. Monthioux and M.J. Hÿtch, "Determining the work function of a carbon-cone cold-field emitter by in situ electron holograph," Micron 63, 2-8, 2014.

[9] J.F. Einsle, C. Gatel, A. Masseboeuf, R. Cours, M.A. Bashir, M. Gunbbins, R.M. Bowman and E. Snoeck "In situ electron holography of the dynamic magnetic field emanating from a hard-disk drive writer," Nano Research 8, 1241-1249, 2015. DOI: 10.1007/s12274014-0610-0

[10] E. Snoeck, F. Houdellier, Y. Taniguchi, A. Masseboeuf, C. Gatel, J. Nicolai, M.J. Hÿtch "Off-axial aberration correction using a B-COR for Lorentz and HREM Modes", Microsc. Microanal., vol. 20 (Suppl 3), pp. $932-$ 933, 2014. DOI:10.1017/S1431927614006382

[11] A. Tonomura "Applications of electron holography", Reviews of Modern Physics, Vol. 59, pp. 639-669, 1987.

[12] Martha R. McCartney, Rafal E. Dunin-Borkowski, David J. Smith "Quantitative measurement of nanoscale electrostatic potentials and charges using off-axis electron holography: Developments and opportunities," Ultramicroscopy, Vol. 203, pp. 105-118, 2019.

[13] K. Harada, A. Tonomura, Y. Togawa, T. Akashi, and T. Matsuda "Double-biprism electron interferometry,"
Appl. Phys. Lett., vol. 84, pp. 3229-3231, 2004. DOI: 10.1063/1.1715155

[14] C. Gatel, J. Dupuy, F. Houdellier, M.J. Hÿtch "Unlimited acquisition time in electron holography by automated feedback control of transmission electron microscope," Appl. Phys. Lett., vol. 113, pp. 133102, 2018. DOI: 10.1063/1.5050906

[15] V. V. Volkov, M. G. Han, Y. Zhu "Double resolution electron holography with simple Fourier transform of fringe-shifted holograms," Ultramicroscopy, vol. 134, pp.175-184, 2013. DOI: 10.1016/j.ultramic.2013.06.018

[16] HREM Research Inc., HoloDark for DigitalMicrograph. https://www.hremresearch.com/holodark/ 